\title{
Parole rapportée et positionnement discursif dans la presse américaine : analyse de l'utilisation des citations dans des commentaires politiques
}

\section{Caroline Peynaud}

\section{(2) OpenEdition Journals}

Édition électronique

URL : http://journals.openedition.org/asp/3047

DOI : $10.4000 /$ asp.3047

ISBN : 978-2-8218-0416-6

ISSN : 2108-6354

\section{Éditeur}

Groupe d'étude et de recherche en anglais de spécialité

\section{Édition imprimée}

Date de publication : 1 mars 2011

Pagination : 43-64

ISSN : 1246-8185

\section{Référence électronique}

Caroline Peynaud, «Parole rapportée et positionnement discursif dans la presse américaine : analyse de l'utilisation des citations dans des commentaires politiques », ASp [En ligne], 59 | 2011, mis en ligne le 04 octobre 2012, consulté le 02 novembre 2020. URL : http://journals.openedition.org/asp/3047 ; DOI : https://doi.org/10.4000/asp.3047

Ce document a été généré automatiquement le 2 novembre 2020.

Tous droits réservés 


\title{
Parole rapportée et positionnement discursif dans la presse américaine : analyse de l'utilisation des citations dans des commentaires politiques
}

\author{
Caroline Peynaud
}

\section{Introduction}

1 Le discours journalistique a pour but d'informer ou de commenter des événements, qu'il s'agisse d'actes ou de paroles, sur lesquels il s'appuie pour se construire. La présence d'éléments extérieurs dans le discours est l'une de ses caractéristiques fondamentales, comme le remarque Sophie Moirand (2007:45):

Le texte journalistique devient alors une mosaïque de voix, constituée d'une pluralité de fils intertextuels, et le fil horizontal du discours apparaît, dans sa matérialité même, fracturé par des marques de cette hétérogénéité.

2 L'hétérogénéité du texte journalistique se fonde sur l'intégration de discours ou d'idées qui lui sont extérieurs. Par ailleurs, le discours journalistique répond à un ensemble de normes; c'est un discours codé qui est soumis à de nombreuses contraintes. Dans ces conditions, il est intéressant de se demander comment les éléments extérieurs s'intègrent dans ces normes, quel est leur rôle dans le discours et en quoi ils l'influencent.

3 Pour comprendre ce processus d'intégration, nous avons choisi de nous appuyer sur un événement de parole, le discours du président Barack Obama sur la réforme de l'assurance maladie devant le Congrès le 9 septembre 2009. Ce discours a été très souvent repris par la presse, expliqué et commenté ; nous avons sélectionné trente et un articles issus de cinq journaux différents (The New York Times, The Washington Post, Los Angeles Times, Chicago Tribune et The Wall Street Journal), quotidiens nationaux et régionaux à diffusion élevée, afin de comprendre de quelle manière cet événement a 
été repris par la presse. S'agissant d'un discours, il est intégré dans les articles sous forme de citations; nous nous sommes donc intéressée à la place des citations dans les articles du corpus, à la fois des citations directes du discours, et des citations émanant de commentateurs extérieurs, et postérieures au discours de B. Obama.

Nous prenons pour hypothèse que les citations, en tant que discours extérieur intégré dans le discours journalistique, ne répondent pas à ses normes, en particulier à celle de l'objectivité que doit refléter un texte journalistique (Charaudeau 2006:4), et qui fait l'objet d'une étude dans le présent article. Le premier but de cet article est donc de montrer quelles sont les différences entre le discours émanant du journaliste, d'une part, et la parole rapportée par le journaliste, d'autre part. Dès lors que les citations sont ajoutées à l'article, elles font néanmoins partie intégrante du discours journalistique et font l'objet d'un processus d'adaptation qui leur permet d'être intégrées dans ce discours. Nous nous demandons donc de quelle manière la citation est modifiée par cette adaptation et dans quelle mesure l'intégration de ce discours autre reflète un souci d'objectivité. Même si les citations ne semblent pas répondre à ce critère, il apparaît qu'il n'y a pas d'antinomie entre présence de citations et objectivité.

Dans la première section, nous dressons un état des lieux des citations dans les textes du corpus, puis nous procédons à une comparaison entre le discours encadrant et le discours encadré. Nous appelons discours encadrant le discours produit par le journaliste car il constitue un cadre pour les citations que nous appelons discours encadré. Cette comparaison est mise en œuvre à travers l'étude des pronoms, des locuteurs premiers et des connecteurs logiques implicites ou explicites, afin de montrer que les citations participent d'un processus d'« effacement énonciatif » (Adam \& Lugrin 2006). Enfin, la dernière section est consacrée à la manière dont le discours encadré est intégré dans le discours encadrant, ce que nous étudions par le biais du choix des citations, des reformulations et de l'orientation de la lecture par les éléments qui encadrent les citations. Il s'agit de comprendre de quelle manière le journaliste utilise les citations pour exprimer son propre point de vue de manière implicite.

\section{1. État des lieux des citations dans les articles}

Nous considérons que les citations qui ont été sélectionnées et intégrées dans l'article en constituent un élément à part entière. C'est d'ailleurs ainsi que le journaliste les utilise dans sa démonstration. Cependant, les citations constituent une partie spécifique du discours journalistique puisqu'elles n'émanent du journaliste qu'en deuxième étape, l'énoncé ayant été produit par un locuteur premier autre que le journaliste, qui se trouve alors en position de locuteur second.

\subsection{Deux types de citations : citations primaires et secondaires}

7 Nous appelons citations primaires toutes les paroles incluses dans le discours de B. Obama du 9 septembre 2009 et reprises dans les articles du corpus. Celles-ci comprennent donc les citations de paroles prononcées par B. Obama dans son discours, mais également les citations d'une lettre écrite par Robert Kennedy avant sa mort et que B. Obama cite, ainsi que l'intervention de Joe Wilson, membre républicain de la chambre des représentants qui, alors que B. Obama explique que la réforme ne s'appliquera pas aux immigrants illégaux, l'interrompt en criant «you lie!» à son 
adresse. Il s'agit d'une citation primaire au sens où elle fait partie du discours-source (cette intervention est incluse dans les retranscriptions ${ }^{1}$ ).

Les autres citations présentes dans le corpus sont appelées citations secondaires, c'està-dire qu'elles émanent de sources autres que l'intervention du 9 septembre 2009. Elles incluent les réactions de divers commentateurs, mais également les citations de paroles de Barack Obama ou de Joe Wilson prononcées dans un autre contexte que le discourssource.

Nous dénombrons 108 citations primaires différentes dans les articles, certaines étant reprises plusieurs fois, dans des articles et journaux différents, et 147 citations secondaires différentes. Seuls sept articles ne contiennent pas de citations secondaires, auxquels nous pouvons ajouter $\mathrm{CT}^{2}$, qui n'en contient qu'une seule, quelque peu décalée par rapport au sujet de l'article. Par ailleurs, cet article, tout comme deux autres de cette catégorie (LAT2 et LAT3), ne contient aucune citation primaire.

10 Les articles sans citations secondaires peuvent être classés dans deux catégories. Il s'agit soit de textes qui n'expriment pas de point de vue particulier sur le discours, des résumés des points principaux par exemple (LAT2, LAT3, NYT6 ou WSJ7), soit de textes dans lesquels le journaliste, au contraire, affirme son point de vue de manière très marquée. C'est le cas de WSJ3, où l'article s'ouvre sur une photographie du journaliste, affirmant ainsi l'importance de la personne de l'auteur, ou de CT3, le seul rédigé à la première personne. Nous pouvons d'ores et déjà postuler une corrélation, que nous tenterons de définir par la suite, entre expression d'un point de vue et utilisation des citations.

\subsection{Les modalités de reprise : parole montrée et parole représentée}

11 Pour traiter de la façon dont la parole est reprise par les journalistes, nous nous appuyons sur la théorie proposée par Jacqueline Authier-Revuz (2004) qui considère que la «représentation du discours autre» ne peut pas se définir par des formes grammaticales, car aucune de ces formes ne lui est propre. Pour définir la représentation du discours autre (RDA), elle s'appuie alors sur deux distinctions. La première se situe entre la prédication concernant le discours autre, lorsqu'il constitue le thème de l'énoncé, et la modalisation du dire par le discours autre, lorsque le thème de l'énoncé est autre, mais que l'énonciateur parle «d'après le discours autre ». La seconde distinction est faite entre une image du discours autre construite par paraphrase et une image construite par monstration (autonymie). L'auteur distingue ainsi quatre zones (voir tableau 1).

Tableau 1. Les quatre zones de la RDA selon J. Authier-Revuz (2004: 41)

\begin{tabular}{|l|l|l|}
\hline & Prédication & Modalisation \\
\hline paraphrase & $\begin{array}{l}\text { Zone 1: zone du discours indirect } \\
\text { (DI) au sens large (« il a annoncé } \\
\text { son retour ) (Authier-Revuz 2004: } \\
41)\end{array}$ & $\begin{array}{l}\text { lone 2: Zone de la modalisation du dire } \\
\text { comme discours second («selon lui les } \\
\text { statistiques mentent ») (Authier-Revuz 2004: } \\
41)\end{array}$ \\
\hline
\end{tabular}




\begin{tabular}{|l|l||l|}
\hline autonymie & $\begin{array}{l}\text { Zone 3: zone du discours direct } \\
\text { (DD) }\end{array}$ & $\begin{array}{l}\text { Zone } 4: \text { Zone de la modalisation autonymique } \\
\text { d'emprunt ("il lui 'compte fleurette' comme } \\
\text { aurait dit ma grand-mère ") (Authier-Revuz } \\
2004: 41)\end{array}$ \\
\hline
\end{tabular}

Partant de cette division en zones, nous avons établi un classement des citations présentes dans le corpus.

13 Les zones 1 et 2 décrivent la représentation paraphrastique du discours autre. Le corpus ne contient aucune occurrence de la zone 2. En revanche, il contient de nombreuses occurrences de la zone 1 , subdivisées en discours indirect (23 occurrences, uniquement de citations primaires), discours indirect libre ( 3 occurrences : 1 primaire et 2 secondaires) et discours narrativisé (36 occurrences: 23 primaires et 13 secondaires).

Il faut noter que les citations au discours indirect, où la citation est intégrée grammaticalement au discours citant, ne sont jamais des citations exactes lorsqu'on les compare au discours-source : dans les occurrences du corpus, le journaliste ne rapporte jamais fidèlement les propos du locuteur premier. J. Authier-Revuz (2004 : 50) explique que la non-adéquation au message original n'influence en rien la catégorie de RDA, et c'est la raison pour laquelle ces citations ont été classées comme discours indirect, mais cette non-adéquation a néanmoins un intérêt pour notre étude. Cette question est traitée dans la section 3.2.

La présence massive de discours narrativisés nous semble également significative. Dans le discours narrativisé, le discours cité s'intègre totalement dans le discours citant.

(1) He talked about this being a moral imperative, an issue of social justice.

And believe me, that resonated. (LAT4)

Dans l'exemple (1), la parole n'est pas autonome, mais intégrée dans celle du journaliste et modifiée par lui. Il s'agit principalement de citations primaires, pour lesquelles le texte-source est disponible pour le grand public et donc pour lesquelles le journaliste pourra plus facilement justifier son intervention pour modifier la citation, puisqu'il fait un commentaire de ce texte. Sur les treize citations secondaires au discours narrativisé, douze sont associées à une représentation autonymique par laquelle le journaliste justifie et légitime son intervention dans le discours narrativisé. Nous développons ce commentaire dans la section 3.3.

17 Les zones 3 et 4 sont celles de la représentation autonymique du discours autre. Nous trouvons un nombre plus élevé d'occurrences pour ces zones, notamment parce que le discours direct (DD) représente la grande majorité des citations: nous relevons 40 occurrences de citations de ce type dans le discours primaire et 116 citations secondaires. Le DD se caractérise par la présence de guillemets pour la phrase entière et d'un verbe introducteur. Patrick Charaudeau (2005: 134) le définit ainsi : « le discours est reproduit tel quel, avec une certaine autonomie. Le locuteur primaire est souvent précisé ». Ce type de citations permet donc de présenter la parole rapportée comme une image fidèle des propos du locuteur premier.

Dans la même catégorie, nous relevons cinq occurrences de discours direct libre, qui sont toutes des citations primaires. Dans cette forme, comme dans le discours direct, la parole rapportée est entourée de guillemets, mais elle peut contenir des marques de 
subordination et, éventuellement, un changement dans les pronoms. Cette forme est donc relativement autonome par rapport au discours encadrant, mais elle est plus intégrée que le discours direct. Nous relevons par exemple :

(2) Perhaps Mr. Obama's most remarkable sleight-of-hand was his claim that he "will not stand by as the special interests use the same old tactics to keep things exactly the way they are." (WSJ4)

Enfin, la zone 4 représente la modalisation autonymique d'emprunt, sous la forme d'îlots textuels, dont nous trouvons seize occurrences de citations primaires et seize secondaires. P. Charaudeau et D. Maingueneau (2002: 190-194) parlent de «discours allusion", précisant que ces segments sont proches du discours direct, mais sont caractérisés par l'absence de verbe introducteur et par une syntaxe incomplète qui force leur intégration syntaxique dans le discours encadrant. Nous trouvons ainsi :

(3) President Obama confronted a critical Congress and a skeptical nation on Wednesday, decrying the "scare tactics" of his opponents. (NYT2)

Nous suivons pour notre part Greta Komur (2004), qui s'appuie sur J. Authier-Revuz (1996) pour donner à ces segments la dénomination d'« îlots textuels». Elle explique qu'ils constituent un processus d'emprunt et qu'ils permettent de rapporter la parole d'autrui tout en s'en distanciant (Komur 2004 : 54). Ils sont définis comme une prise de distance du journaliste par rapport aux mots venus d'ailleurs, ou comme :

[...] une sorte de cryptodialogue intérieur à une seule énonciation où l'énonciateur a le beau rôle: c'est lui qui observe, s'amuse, se moque, s'indigne des paroles de

l'autre. (Rey-Debove 1997 : 266)

21 Le journaliste se dégage de toute responsabilité quant au contenu de la séquence représentée, mais possède des moyens pour « influencer notre interprétation par le jeu des adverbes, des adjectifs, des verbes introducteurs » (Komur $2004: 61$ ). Il s'agit d'une forme très courante dans notre corpus, en particulier pour des expressions comme public option ou security and stability, qui sont reprises de manière préférentielle sous forme d'îlots textuels. L'utilisation de cette forme est due au fait que B. Obama n'utilise la première expression par exemple qu'entourée de beaucoup de précautions, pour se prémunir contre toute attaque sur ce sujet très controversé. Or, les journalistes ne prennent pas tant de précautions dans leur discours et l'expression est utilisée en tant que telle, sans explication. On assiste à une simplification du propos du discours-source qui s'appuie sur l'utilisation d'îlots textuels.

Tableau 2. Tableau récapitulatif du nombre de citations de chaque zone dans le corpus

\begin{tabular}{|c|c|c|c|c|c|}
\hline & Zone 1 & Zone 2 & Zone 3 & Zone 4 & TOTAL \\
\hline Citations primaires & $\begin{array}{l}\text { DI }: 23 \\
\text { DIL }: 1 \\
\text { DN }: 23\end{array}$ & 0 & $\begin{array}{l}\text { DD : } 40 \\
\text { DDL }: 5\end{array}$ & IT $: 16$ & 108 \\
\hline Citations secondaires & $\begin{array}{l}\text { DIL }: 2 \\
\text { DN }: 13\end{array}$ & 0 & DD : 116 & IT $: 16$ & 147 \\
\hline TOTAL & 62 & 0 & 161 & 32 & 255 \\
\hline
\end{tabular}



représentations autonymiques sont en général employées pour orienter la compréhension de la citation par le lecteur. Les premières donnent une information que les secondes semblent illustrer. Ce constat remet en question l'idée de la parole autonyme comme prise de distance, puisque la parole autonyme n'est pas utilisée seule. Il semblerait plutôt qu'il s'agisse d'une justification ou de la légitimation du contenu des représentations paraphrastiques (formes où le journaliste intervient le plus) par l'autonymie (formes où il n'intervient pas, et où son discours est donc plus légitime puisqu'il s'appuie sur la parole d'autrui).

27 Partant de ce constat, nous cherchons à comprendre comment les citations remplissent cette fonction de légitimation et dans quelle mesure elles permettent au journaliste d'exprimer des opinions qu'il ne pourrait pas exprimer dans son propre discours.

\section{2. Étude comparée du discours encadrant et du discours encadré : les citations comme lieu privilégié d'expression du point de vue}

28 À travers cette comparaison, nous souhaitons montrer que les caractéristiques du discours encadré diffèrent de celles du discours encadrant. Le journaliste respecte certaines normes dans son discours, mais ces normes ne s'appliquent pas nécessairement aux citations. Nous observons ainsi dans le discours encadrant un effacement de la personne du journaliste, qui va de pair avec une légitimation du contenu de l'article, une apparence d'objectivité étant créée à travers l'emploi des citations. 


\subsection{Les citations au sein d'une stratégie d'effacement énonciatif}

La comparaison entre discours encadré et discours encadrant a conduit à l'étude des pronoms de première personne dans les différentes strates du discours. Il apparaît que le discours encadré contient plus de marqueurs de personnalisation que le discours encadrant. Ce déséquilibre s'explique par le rôle même des locuteurs, puisque B. Obama, dans son discours, s'engage personnellement à réaliser la réforme et que les locuteurs secondaires interviennent en tant que commentateurs subjectifs.

Les pronoms de première personne sont absents du discours encadrant, alors qu'ils sont très fréquents dans les citations primaires. Le discours de B. Obama contient beaucoup d'occurrences de I (57) et de we (69) (respectivement 10,2 \%o et 12,4 $\%^{3}$ ). Les I réfèrent toujours à $\mathrm{B}$. Obama, et les we renvoient généralement à tous les Américains, en incluant le président lui-même. Dans les citations du discours retenues dans les articles, les pronoms sont par ailleurs associés à des verbes exprimant une volonté marquée (will, want : 25 , soit $20 \%$ des citations primaires), à des modaux ou semimodaux (need, have to, can : 19, soit $15 \%$ ) ou à des procès intellectuels (know, understand, believe... : 32, soit $25 \%$ ). Nous observons donc dans ce discours une forte personnalisation.

31 Le même constat est valable pour les citations secondaires où les pronoms personnels de première personne sont fréquents : 45 citations sur 116 contiennent des $I$, soit un peu plus d'un tiers, et nous comptons 58 occurrences de we, utilisé lorsque l'énonciateur représente une communauté. Les we sont associés à des modaux ou semimodaux (dont les plus courants sont ought to, need, have to, should, avec un sens prescriptif majoritaire : 20 , soit $34,5 \%$ des citations contenant we), ou à des procès intellectuels (think, talk, heard, understand: 14 , soit $24,1 \%$ ). Les I sont associés à des procès intellectuels, marques qui indiquent que l'énonciateur affirme son opinion (think, believe, I'm a big believer, guess, know : 46 occurrences, représentant $72 \%$ des citations, avec une prédominance de I think / thought : 23 occurrences). Nous observons clairement une mise en avant de la subjectivité du locuteur.

À l'inverse, les I et we sont très peu représentés dans le discours encadrant, seul un article du corpus y a recours en dehors des citations. Cet article (СТ3) est d'ailleurs quelque peu atypique par le ton de son auteur et par son contenu. Le discours encadrant élimine presque les pronoms de première personne, pour ne garder que ceux de la troisième personne : 209 occurrences de it et 334 occurrences de he. Le discours journalistique semble donc s'appuyer sur une objectivation du discours et sur un effacement énonciatif dû au fait que le journaliste ne s'affirme pas dans sa position d'énonciateur dans le texte encadrant, c'est-à-dire celui qu'il prend à son compte. Les modaux employés dans un sens radical sont absents, ainsi que les verbes marquant l'expression d'une opinion (think, believe...).

Tableau 3. Tableau récapitulatif des pronoms de première personne et de leurs associations dans les différents types de discours ${ }^{4}$

\begin{tabular}{|l|l|l|l|l|l|}
\hline & (1) I & (2) We & $\begin{array}{l}\text { (3) Associés } \\
\text { majoritairement } \\
\text { à }\end{array}$ & $\begin{array}{l}\text { (4) Nombre et } \\
\text { pourcentage }\end{array}$ & $\begin{array}{l}\text { (5) } \\
\text { Nombre } \\
\text { total de } \\
\text { mots }\end{array}$ \\
\hline
\end{tabular}




\begin{tabular}{|c|c|c|c|c|c|}
\hline $\begin{array}{l}\text { Discours de } \\
\text { B. Obama }\end{array}$ & $\begin{array}{l}57 \text { occurrences } \\
10,2 \% \text { o }\end{array}$ & $\begin{array}{l}69 \\
\text { occurrences } \\
12,4 \% \text { o }\end{array}$ & $\begin{array}{l}\text { - Verbes } \\
\text { exprimant la } \\
\text { volonté } \\
\text { - Modaux ou semi- } \\
\text { modaux } \\
\text { - Procès } \\
\text { intellectuels }\end{array}$ & $\begin{array}{l}25(20 \%) \\
19(15 \%) \\
32(25 \%)\end{array}$ & 5563 \\
\hline $\begin{array}{l}\text { Citations } \\
\text { secondaires }\end{array}$ & $\begin{array}{l}45 \text { occurrences } \\
7,5 \% \text { o }\end{array}$ & $\begin{array}{l}58 \\
\text { occurrences } \\
9,7 \% \text { o }\end{array}$ & $\begin{array}{l}\text { - Modaux ou semi- } \\
\text { modaux } \\
\text { - Procès } \\
\text { intellectuels }\end{array}$ & $\begin{array}{l}20(34,5 \%) \\
60(58 \%)\end{array}$ & 5991 \\
\hline $\begin{array}{l}\text { Discours } \\
\text { encadrant }\end{array}$ & $\begin{array}{l}7 \text { occurrences } \\
\text { (dans un seul } \\
\text { article) } \\
0,23 \% \text { o }\end{array}$ & 0 & - Verbes d'action & $5(71,5 \%)$ & 29427 \\
\hline
\end{tabular}

Tandis que le journaliste refuse d'exprimer son opinion de manière personnalisée et produit un discours encadrant qui se présente comme objectif, c'est-à-dire focalisé sur l'objet, il a recours aux citations des deux sortes, seules parties du discours contenant des marques de subjectivité, dans une logique de légitimation de l'opinion, puisqu'on ne pourra pas lui reprocher d'exprimer des opinions si elles sont attribuées à d'autres locuteurs.

Cette remarque est cohérente avec l'emploi des marqueurs évaluatifs dans les citations, par opposition au discours encadrant. Nous définissons les marqueurs évaluatifs comme «l'ensemble des marques par lesquelles l'énonciateur exprime un jugement de valeur ou une réaction affective" (Charaudeau \& Maingueneau 2002: 51). Celles-ci incluent toutes les catégories grammaticales, avec une prédominance des adjectifs et des adverbes dans notre corpus. Soixante-neuf pour cent de l'ensemble des citations secondaires relevées contiennent des marqueurs évaluatifs, alors qu'il n'y en a pas dans le discours encadrant. L'utilisation des marqueurs évaluatifs confirme l'idée que les journalistes recourent aux citations pour exprimer un point de vue et le défendre, de manière masquée, puisqu'ils essaient le plus souvent de maintenir en apparence un équilibre entre les points de vue.

\subsection{Les locuteurs premiers comme figures d'autorité}

La section précédente a conclu à une mise en avant de la subjectivité du locuteur dans les citations. Dès lors, l'identité du locuteur est primordiale et une catégorisation des locuteurs premiers permet d'observer que le journaliste choisit ses sources et les introduit avec soin afin de les présenter systématiquement comme des figures d'autorité.

Les citations du discours de B. Obama constituent clairement une légitimation car le journaliste montre qu'il s'appuie sur des faits, sur ce qui a réellement été dit pendant le discours et qui justifie son commentaire. Pour les citations secondaires, ce constat demande à être développé. Le journaliste s'appuie sur les locuteurs premiers pour 
analyser les faits et faciliter la compréhension du lecteur. Il est donc important que ces éléments paraissent légitimes. C'est pour cette raison qu'ils sont identifiés d'une manière très claire qui permet au journaliste de montrer en quoi ils constituent des figures d'autorité. Nous avons relevé six catégories de locuteurs premiers.

La première catégorie est celle des énonciateurs primaires (B. Obama et J. Wilson), qui interviennent dans des contextes différents, le plus souvent en rapport avec les excuses que prononce J. Wilson à la suite de son intervention. Nous relevons douze citations de ce type, dont dix concernent les excuses de J. Wilson à B. Obama. Ces derniers ne sont pas réellement commentateurs du discours, puisque c'est leur discours en lui-même qui constitue l'événement. Leur rôle est donc particulier au sein des locuteurs et la question de leur légitimité ne se pose pas.

Nous relevons également 45 interventions de spectateurs du discours primaire qui le commentent, avec une récurrence de certains intervenants (McCain [3], House Minority Leader John Boehner [3], par exemple), qui composent la deuxième catégorie. Il semble que la plupart des journaux puisent à des sources similaires. Ces intervenants sont toujours identifiés avec le nom, la fonction, le parti, l'État et éventuellement la position sur la réforme, si elle ne suit pas la ligne du parti ou s'ils ont un rôle particulier dans la réforme, ce qui permet de montrer en quoi ils sont compétents pour commenter le discours.

La troisième catégorie est celle des officiels non spectateurs qui n'ont pas assisté au discours (14 citations). Comme pour la catégorie précédente, chaque élément ajouté précise soit leur position sur la réforme, soit la raison de leur légitimité à s'exprimer sur ce sujet.

(6) Maya MacGuineas, president of the Committee for a Responsible Federal Budget, which is supported by business. (NYT8)

Les éléments de description sont les mêmes pour la quatrième catégorie, celle des parties prenantes (15), qui comprend des présidents ou porte-parole d'associations, ou des patrons de compagnies d'assurances. Leur légitimité est donc justifiée de manière similaire.

41 La cinquième catégorie est celle des experts (7 citations) qui peuvent être d'anciens conseillers politiques ou des professeurs de droit. Leur présentation explique systématiquement pourquoi leur opinion est citée, comme c'est le cas dans l'exemple (7) :

(7) Alissa Fox, a senior vice president at the BlueCross Blueshield Association, which represents 39 independent insurers. (WSJ6)

Enfin, une catégorie « autres » comprend 21 citations, dont 16 sont des citoyens ou des patrons de petites entreprises qui réagissent à la réforme. Nous relevons également une référence à un article du New York Times de l'époque du président Clinton (citée dans The New York Times) et une citation non attribuée introduite par the allegation that... Les exemples où les locuteurs premiers sont des citoyens sont éclairants dans la mesure où il s'agit d'une légitimation faisant appel à l'expérience : ces citoyens ont une légitimité parce qu'ils vivent la situation de l'intérieur. Il y a de nombreuses citations de ce type, toutes rassemblées dans quelques articles seulement : NYT9, WP2, WSJ2, WSJ5 et WSJ8. Peut-être ce procédé est-il employé dans The Wall Street Journal car ce journal a une orientation économique assez éloignée des préoccupations des familles modestes qui 
s'inquiètent de la réforme ${ }^{5}$. Le fait de citer des citoyens modestes apporte donc un point de vue différent sur la question et une légitimité supplémentaire lorsque le journal aborde les problèmes que pourrait poser la réforme.

Tous les éléments de présentation des locuteurs secondaires ont pour but de légitimer ces locuteurs et par conséquent les propos des journalistes, mais cette légitimation n'intervient qu'en seconde étape et de manière implicite. La section suivante montre que l'implicite est une caractéristique récurrente du discours journalistique.

\subsection{L'explicite du discours encadré et l'implicite du discours encadrant}

L'explication et l'argumentation explicite se trouvent uniquement dans les citations et très peu dans le discours encadrant. Le discours journalistique n'est pas présenté comme un discours argumentatif, mais il est d'abord argumentatif au sens où les informations contenues dans l'article sont sélectionnées pour servir le point de vue du journaliste. La citation, ainsi sortie de son contexte immédiat, prend un sens parfois détourné et devient un élément de l'argumentation de l'article. Vignaux (1981: 91) explique ainsi qu' " énoncer, cela revient à argumenter, du simple fait qu'on choisit de dire et d'avancer certains sens plutôt que d'autres ». En tant que "troisième homme ", le rôle du journaliste est bien de prendre position pour éclairer le lecteur dans sa compréhension des problèmes.

Précisant cette idée, Blandine Pennec explique que l'expression de la causalité, définie comme «lien logique établi entre les événements et permettant d'expliquer l'un d'eux » (2010: 34) est toujours implicite dans les articles de presse. Elle distingue les marqueurs implicites (segments à valeur temporelle, propositions relatives, et propositions non finies) et explicites (because, as, since et for) d'expression de la cause. Elle remarque également que « d'une connexion logique découle fréquemment l'idée de mise en cause ». B. Pennec relève dans son corpus journalistique de nombreuses occurrences de because, la quasi-absence des autres marqueurs et la fréquence remarquable de marqueurs implicites. Elle conclut que « dans ces énoncés, la notion de cause est en fait suggérée par l'énonciateur et reconstruite interprétativement par le co-énonciateur » (Pennec $2010: 45)$.

L'analyse de notre corpus montre que les remarques de B.Pennec s'appliquent uniquement au discours encadrant, alors que les résultats sont tout à fait différents pour les citations. Cette double caractéristique est cohérente avec le fait que des locuteurs tels que B. Obama ou que les locuteurs secondaires interviennent dans la presse pour donner un point de vue et l'affirment donc de manière très claire. Nous trouvons ainsi 19 occurrences de because dans l'ensemble du corpus, dont 18 se trouvent dans des citations, primaires ou secondaires. Même le marqueur le plus courant de la causalité selon B. Pennec, c'est-à-dire because, n'apparaît que de manière marginale dans le discours encadrant dans notre corpus.

47 Afin d'approfondir l'analyse, nous avons relevé tous les marqueurs argumentatifs se trouvant dans les citations et les avons comparés à ceux présents dans le texte encadrant. Nous trouvons ainsi dans les citations de nombreux éléments tels que des marques de la subjectivité de B. Obama (I have to say that, I believe, and I have no doubt, let me discuss, unfortunately) et de l'intersubjectivité (you see), des connecteurs logiques qui visent à rendre le discours cohérent et donc convaincant (but, then, finally, while, thanks 
to, as, now, despite all this, for example, in fact, because, add it all up, and...), ou des appels à la logique et au bon sens. Dans les citations secondaires, nous relevons également: because (4), but (38), if (16), while (6 de sens logique), in fact (2), ou then (2). Après comparaison, il apparaît qu'aucun de ces marqueurs n'est employé, du moins dans un sens argumentatif, dans le texte encadrant.

Ainsi, dans notre corpus, seul le discours encadrant correspond aux remarques de B. Pennec (2010), tandis que les citations sont clairement argumentatives. Par le truchement des citations, les articles contiennent bien des marqueurs d'argumentation explicite, mais tout en dédouanant le journaliste, puisqu'il n'a qu'un rôle d'intermédiaire dans ce discours. Il semble que le journaliste ne prenne jamais la responsabilité des explications qui figurent dans son article. Ce phénomène ferait donc partie de la stratégie d'effacement énonciatif mise en place dans le discours de la presse.

Nous avons vu que discours encadré et discours encadrant ont des caractéristiques différentes, la subjectivité et l'autorité du locuteur étant mises en avant par le biais des citations afin de légitimer le discours encadrant. Ces deux types de discours font néanmoins partie du même ensemble et la question de l'intégration de l'un dans l'autre est essentielle pour comprendre comment le journaliste intègre les citations dans la construction de son propre discours.

\section{L'intégration du discours encadrant dans le discours encadré : l'intervention du journaliste dans la sélection et la mise en forme des citations}

50 Nous défendons ici l'idée que le journaliste a recours aux citations comme vecteur de son point de vue. Les citations étant un procédé de légitimation, elles permettent au journaliste de rendre son point de vue moins contestable et de l'exprimer de manière moins visible.

\subsection{Le choix des citations : une première marque de point de vue}

51 En étudiant le choix des citations opéré par les journalistes, il apparaît que cette sélection reflète une certaine orientation de la presse et influence la compréhension que le lecteur peut avoir du discours. La presse s'intéresse en effet aux formules qui frappent l'esprit et qui pourront attirer l'attention du lecteur. C'est ainsi qu'elle répond à l'enjeu de captation. William Raspberry, dans le Herald Tribune, (traduction publiée dans le Nouveau Quotidien de Lausanne en 1995) observe que « Dans les médias, le conflit prime toujours sur la substance » (in Lorda $2001: 5$ ). Il semble que ce soit exactement le cas dans ce corpus.

52 La citation la plus reprise ne vient pas de B. Obama, mais c'est le "you lie » (17 occurrences) de Joe Wilson, avec lequel il interrompt B. Obama au milieu de son discours. C'est bien l'exemple même de ce qui a pu représenter un conflit pendant le discours: l'interruption violente du discours par le député a fait scandale dans la presse. Les autres citations primaires qui sont reprises trois fois ou plus dans le corpus apparaissent dans le tableau 4. 
Tableau 4. Citations les plus fréquemment reprises dans le corpus

\begin{tabular}{|c|c|c|}
\hline Citations & $\begin{array}{l}\text { Nombre } \\
\text { d'occurrences }\end{array}$ & Articles \\
\hline $\begin{array}{l}\text { (8) I will not waste time with those who have made the } \\
\text { calculation that it's better politics to kill this plan than improve } \\
\text { it. }\end{array}$ & 4 & $\begin{array}{l}\text { NYT2 } \\
\text { NYT5 } \\
\text { WSJ6 } \\
\text { WP3 }\end{array}$ \\
\hline (9) It is a lie, plain and simple. & 6 & \begin{tabular}{|l} 
NYT2 \\
NYT4 \\
WSJ6 \\
WSJ7 \\
WP3 \\
fois $)$
\end{tabular} \\
\hline $\begin{array}{l}\text { (10) And out of this blizzard of charges and countercharges, } \\
\text { confusion has reigned. }\end{array}$ & 3 & $\begin{array}{l}\text { NYT3 } \\
\text { NYT4 } \\
\text { WP3 }\end{array}$ \\
\hline $\begin{array}{l}\text { (11) And I will not accept the status quo as a solution. Not this } \\
\text { time. Not now. }\end{array}$ & 3 & $\begin{array}{l}\text { NYT4 } \\
\text { WSJ3 } \\
\text { WSJ6 }\end{array}$ \\
\hline $\begin{array}{l}\text { (12) I will not sign a plan that adds one dime to our deficits either } \\
\text { now or in the future. Period. }\end{array}$ & 4 & $\begin{array}{ll}\text { NYT8 } & (2 \\
\text { fois }) & \\
\text { WSJ7 } & \\
\text { CT3 }\end{array}$ \\
\hline $\begin{array}{l}\text { (13) The time for bickering is over, the time for games has passed. } \\
\text { Now is the season for action. }\end{array}$ & 4 & $\begin{array}{l}\text { NYT2 } \\
\text { WSJ6 } \\
\text { WP1 } \\
\text { WP3 }\end{array}$ \\
\hline $\begin{array}{l}\text { (14) I am not the first president to take up this cause, but I am } \\
\text { determined to be the last. }\end{array}$ & 3 & $\begin{array}{l}\text { NYT3 } \\
\text { WP1 } \\
\text { WP3 }\end{array}$ \\
\hline (15) It is only one part of my plan. & 5 & $\begin{array}{l}\text { NYT1 } \\
\text { WSJ6 } \\
\text { CT1 } \\
\text { WP3 } \\
\text { fois })\end{array}$ \\
\hline
\end{tabular}



dans (8), à nouveau le mot lie dans (9) et les termes charges and countercharges dans (10). Les citations (11) (12) (13) et (14) montrent la détermination du président, qui prend des décisions tranchées, et elles soulignent le conflit entre B. Obama et les opposants à la réforme. Enfin, (15) revient sur l'aspect le plus controversé de la réforme, celui de la possibilité d'une assurance maladie publique, que l'on trouve dans les articles sous le nom de the public option. Elle est donc citée pour illustrer cette question, de la même manière que (12) est citée pour réagir sur la question controversée du coût de la réforme. Le choix des citations est donc révélateur de ce qui intéresse la presse, puisque c'est le conflit qui attire le public.

Par ailleurs, dans les citations primaires, les remarques que le lecteur retient principalement sont celles concernant la politique menée par B.Obama; elles contiennent très peu d'informations sur la réforme en elle-même. Le même constat est d'ailleurs valable à propos des citations secondaires : la plupart portent sur la politique de B. Obama, plutôt que sur les détails de la réforme, à l'image de la citation (16) :

(16) "It sounded very much like the Chicago politics that I know he's familiar with," said Arizona Sen. Jon Kyl, the No. 2 Republican in the Senate. "It appeared as if he was trying to ram something through." (LAT4)

Si l'on s'intéresse aux thèmes et au titre des articles, le constat est similaire : il s'agit davantage d'analyses politiques que de descriptions ou de commentaires de la réforme. Les citations qui expliquent précisément la réforme ne sont présentes que dans un nombre réduit d'articles qui expliquent la réforme sans y apporter d'élément d'analyse (LAT2, LAT3, NYT6, ou WSJ7). Le reste des articles - soit la grande majorité - se concentre sur les citations plus polémiques. Ainsi, il apparaît que les citations insistent sur les mêmes points que ceux mis en valeur par les journalistes; il semble que les deux discours répondent aux mêmes critères de sens.

\subsection{Discours indirect et reformulations : l'ambiguïté comme stratégie énonciative}

56 Nous avons mentionné dans la première partie la présence d'un type de discours indirect dont il n'existe pas d'équivalent dans le discours-source. Nous appelons cette forme de discours indirect "discours-reformulation». Il s'agit de 23 citations primaires, sachant qu'il est impossible de distinguer discours-reformulation et discours indirect dans les citations secondaires sans avoir accès aux sources. La principale caractéristique du discours-reformulation est qu'il a toutes les marques du discours indirect et qu'il fait donc partie de cette catégorie du point de vue de la classification appliquée plus haut, mais qu'il n'existe pas de segment équivalent dans le discourssource. Il ne s'agit donc pas de citations textuelles, mais d'une reformulation des propos du locuteur primaire dans laquelle le journaliste peut ajouter des éléments de sa propre interprétation.

Le journaliste a la possibilité de modifier le sens de toutes les citations puisqu'elles sont extraites de leur contexte textuel immédiat et qu'elles peuvent donc être manipulées. Cependant, le discours reformulé constitue un espace de liberté particulier pour deux raisons. D'une part, le journaliste n'est pas tenu de rapporter fidèlement les propos du locuteur premier, puisqu'il ne s'agit pas de discours direct, dans lequel des mots précis 
sont attribués à un locuteur et dont l'adéquation à la source peut être vérifiée. D'autre part, l'ambiguïté entre discours indirect et discours-reformulation donne à ce type de discours une légitimité que le journaliste n'aurait pas s'il s'exprimait sans attribuer ses paroles à un autre énonciateur, puisque le lecteur assimile ces formules à des citations textuelles. Nous avons choisi d'étudier plus précisément certains exemples de discours reformulé afin de tenter de comprendre la raison pour laquelle le journaliste choisit ce procédé pour rapporter la parole, alors que la section 1.2. a montré que la représentation autonymique est celle qui lui donne le plus de légitimité.

Tableau 5. Comparaison entre discours-reformulation et équivalents dans le discours-source

\begin{tabular}{|c|c|c|}
\hline Article & $\begin{array}{l}\text { Citation reformulée dans le discours } \\
\text { journalistique }\end{array}$ & Énoncé d'origine dans le discours-source \\
\hline $\begin{array}{l}(17) \\
\text { NYT1 }\end{array}$ & $\begin{array}{l}\text { As an interim step, Mr. Obama called for } \\
\text { establishing a high-risk pool that could } \\
\text { immediately offer coverage to those who } \\
\text { cannot afford health insurance. }\end{array}$ & $\begin{array}{l}\text { There will be a hardship waiver for those } \\
\text { individuals who still cannot afford coverage. }\end{array}$ \\
\hline $\begin{array}{l}(18) \\
\text { NYT1 }\end{array}$ & $\begin{array}{l}\text { In his speech on Wednesday, the president } \\
\text { tried to make clear that the bar would be } \\
\text { set high enough not to break his anti-tax } \\
\text { pledge. }\end{array}$ & $\begin{array}{l}\text { they argue that these private companies can't } \\
\text { fairly compete with the government. And } \\
\text { they'd be right if taxpayers were subsidizing } \\
\text { this public insurance option. But they won't } \\
\text { be. }\end{array}$ \\
\hline $\begin{array}{l}(19) \\
\text { NYT6 }\end{array}$ & $\begin{array}{l}\text { [...] he accurately stated that the United } \\
\text { States is the only developed democracy } \\
\text { with millions of uninsured citizens with } \\
\text { little or no access to anything but } \\
\text { emergency care. }\end{array}$ & $\begin{array}{l}\text { We are the only advanced democracy on Earth } \\
\text { - the only wealthy nation - that allows such } \\
\text { hardships for millions of its people. There are } \\
\text { now more than thirty million American } \\
\text { citizens who cannot get coverage. }\end{array}$ \\
\hline $\begin{array}{l}(20) \\
\text { NYT9 }\end{array}$ & $\begin{array}{l}\text { Mr. Obama said that his health care } \\
\text { overhaul would not extend benefits to } \\
\text { illegal immigrants. }\end{array}$ & $\begin{array}{l}\text { There are also those who claim that our reform } \\
\text { effort will insure illegal immigrants. This, too, } \\
\text { is false - the reforms I'm proposing would not } \\
\text { apply to those who are here illegally. }\end{array}$ \\
\hline $\begin{array}{l}(21) \\
\text { LAT4 }\end{array}$ & $\begin{array}{l}{[\text { [..] the president's pledge to ensure that }} \\
\text { an overhaul would not add to the } \\
\text { government's debt. }\end{array}$ & $\begin{array}{l}\text { First, I will not sign a plan that adds one dime } \\
\text { to our deficits - either now or in the future. }\end{array}$ \\
\hline
\end{tabular}

Dans chacun de ces exemples, le verbe introducteur est signalé en gras et les reformulations du discours-source en italique. À l'analyse, il apparaît que différents procédés sont à l'œuvre. Nous remarquons tout d'abord la création de "formules " (Krieg-Planque 2006) qui ne sont pas présentes dans le discours-source, mais qui sont souvent reprises par la presse, probablement pour des raisons de concision. C'est le cas de high-risk pool (17), anti-tax pledge (18), ou overhaul (20). La presse évite ainsi les paraphrases explicatives utilisées dans le discours-source. Les reformulations des exemples (19) et (21) répondent à la même recherche de concision, puisqu'un énoncé complexe et très développé dans le discours-source est résumé en une phrase simple. 
La presse semble ici à nouveau opérer une simplification des enjeux présentés dans le discours de B. Obama.

Il apparait également dans ce tableau que les verbes introducteurs et les éléments qui les modifient jouent un rôle important dans l'introduction de la citation par le journaliste, comme c'est le cas avec (19) he accurately stated, ou dans (18) the president tried to make clear. C'est également le cas avec l'utilisation de termes tels que pledge, (18) et (21), qui permettent au journaliste de rappeler une promesse électorale de B. Obama tout en donnant de la force à son argument, sans montrer explicitement qu'il s'implique dans une évaluation du discours. Les éléments qui entourent la citation ont un rôle à jouer dans le message que le journaliste veut transmettre par le biais de la citation.

\subsection{Les ajouts du locuteur second dans le texte encadrant immédiatement les citations : l'orientation de la lecture de la citation}

Le discours journalistique met en œuvre plusieurs procédés pour orienter le lecteur dans sa compréhension de la citation, tout en restant toujours dans l'implicite puisqu'il n'émet pas de jugement explicite sur la citation ou le locuteur premier. Nous avons relevé trois procédés de ce type : les modalités d'insertion des citations dans le texte, les combinaisons entre discours indirect ou discours narrativisé et discours direct, et la présentation du locuteur premier.

61 Nous remarquons une grande similarité dans le choix des verbes introducteurs. La citation de Joe Wilson, « you lie ", est ainsi reprise 17 fois, toujours au discours direct, et introduite généralement par shout (forme nominale ou verbale, 11 occurrences), plus ponctuellement par yell (yell out ou yell back, 3 occurrences), les autres formes sont marginales. Il semble que la manière de reprendre la citation corresponde à la volonté de présenter l'information comme un scandale, ce qui explique les récurrences dans les verbes introducteurs choisis. Le tableau 5 met également cet aspect en lumière. Cependant, le verbe introducteur le plus courant reste de loin say. Les autres éléments qui entourent la citation informent le lecteur sur ce qu'il va lire et ce qu'il devrait comprendre.

Nous avons vu dans la section 1.2. que les combinaisons entre discours indirect ou discours narrativisé et discours direct sont très courantes dans notre corpus. Or, elles remplissent une fonction bien précise, puisque, souvent, le discours indirect ou le discours narrativisé introduit ce qui va être dit en discours direct de manière à préparer le lecteur à ce qu'il va lire. Le lecteur est très rarement surpris en lisant la citation directe. Par exemple :

(23) Republicans also said the heckling was out of line. "I think we ought to treat the president with respect," said Senator Mitch McConnell of Kentucky, the Republican leader, "and anything other than that is not appropriate." (NYT3)

Dans ce cas, le discours indirect que nous avons écrit en gras reprend la même idée que celle exprimée au discours direct, avec la répétition entre out of line et not appropriate. Peut-être le fait d'exprimer deux fois le même sens permet-il au journaliste de s'assurer que son message sera bien compris, comme dans l'exemple (24): 
(24) Underscoring tensions among Capitol Hill Democrats, House Ways and Means Committee Chairman Charles B. Rangel (D-N.Y.) - a key architect of the House healthcare bill - derided the Senate's legislative effort. "I wouldn't spend a lot of time on what the Senate is thinking. They are not thinking, quite frankly," Rangel said. "One thing we don't have responsibility [for] is to check with the other body." (LAT4)

64

\section{au discours direct. Le verbe deride est par ailleurs un jugement sur ce que dit le locuteur premier; il en donne une interprétation particulière qui influence ce que le lecteur comprend de la citation. Ce procédé est parfois utilisé de manière inversée : \\ (25) "It's not critical that the president lock down every single outstanding question right now," said Chris Jennings, a senior health care adviser in the Clinton administration. "In fact, going too far in one way or the other might actually mess things up." There are risks to the White House strategy. (CT27)}

Ici, la citation est suivie d'un résumé dont il est difficile de savoir s'il doit être attribué au journaliste ou au locuteur premier. Le résumé donne une interprétation de la citation. Ces combinaisons permettent donc au journaliste de ne pas présenter les citations au discours direct comme des faits bruts offerts à l'interprétation du lecteur, mais au contraire de les insérer dans son texte en les commentant de manière implicite.

Enfin, les journalistes s'appuient sur leur manière de présenter le locuteur premier pour orienter l'interprétation de la citation par le lecteur. Les informations données sur le locuteur premier constituent une indication sur le contenu de la citation et c'est pour cette raison que ces informations sont souvent très développées. Par exemple :

(26) Senator Bob Corker, a Tennessee Republican who has indicated he would like to work with Democrats on health care, said: "The point of the speech was to unify the president's Democratic base, not to advance policy. We heard a lot of pontifications and platitudes." (NYT7)

Ici, le fait que le locuteur soit républicain induit une opinion négative sur le discours lui-même, mais l'information who has indicated he would like to work with Democrats on health care laisse également attendre une volonté politique de voir aboutir le projet. Ces deux éléments sont présents dans la citation. Les exemples où il s'agit de citoyens montrent également ce type de procédé :

(27) Jeremy Schmidt, a married 30-year-old with a goatee and a red-andblue tattoo - "Welcome to Las Vegas" - on his right forearm said he worried about his stepfather, a loading dock supervisor who got a triple bypass last year. "If he gets too sick to do his job he'll lose his insurance," Mr. Schmidt said. "That's a huge concern for my family." (WSJ2)

Dans l'exemple (27), la description du locuteur constitue un «effet de réel»; la description physique fait imaginer une personne d'un milieu social modeste et la citation qui suit présente bien des préoccupations de tous les jours. La description produit également un effet comique : le lecteur, tout comme le journaliste, ne peut pas s'identifier à ce locuteur. Dans cet exemple, le journaliste fait appel à un ensemble de valeurs qu'il pense partager avec le lecteur pour orienter sa lecture. La présentation du locuteur permet donc d'orienter la compréhension de la citation au sens où le lecteur 
s'attend à un certain type de contenu, et il est rare que la citation ne corresponde pas au sens attendu.

\section{Conclusion} de très nombreux types de citations et nous remarquons que le choix du type de discours rapporté employé correspond à la recherche d'un équilibre entre expression d'une opinion et maintien d'une apparence d'objectivité dans les articles. Les citations ont donc un rôle bien précis à jouer dans les articles.

En raison de la fonction particulière des citations, leur contenu a des caractéristiques singulières. Nous avons ainsi remarqué la prédominance de procédés explicites dans le discours encadré tranchant avec l'implicite du discours encadrant. Les citations ont, en elles-mêmes, plus de légitimité et le fait qu'elles émanent d'intervenants extérieurs dispense le journaliste d'avoir à justifier leur contenu ou de rechercher une apparence d'objectivité. Or, le journaliste s'appuie sur la légitimité intrinsèque des citations pour transmettre un message correspondant à ses propres valeurs, que ce soit en modifiant la citation (reformulations), ou en orientant son interprétation (verbes introducteurs et combinaisons d'autonymie et de paraphrase) et bien sûr par son choix même des citations. L'utilisation de citations dans le discours journalistique n'est donc pas un procédé neutre, mais elle permet au journaliste d'exprimer des idées bien précises, au risque parfois de détourner le sens de la citation. C'est pour cette raison que, si les citations ne correspondent pas exactement aux caractéristiques du discours journalistique, elles font néanmoins partie intégrante de l'écriture journalistique.

71 Cet article a notamment permis de montrer que le discours journalistique est caractérisé par une hétérogénéité intrinsèque qu'il est impossible de réduire linguistiquement, puisque les deux discours restent fondamentalement hétérogènes du point de vue linguistique. Nous nous sommes seulement intéressée ici à une forme particulière d'intégration d'un discours extérieur dans le discours journalistique à travers les citations, mais il serait nécessaire de poursuivre en étudiant des exemples différents de discours autres influençant le discours journalistique, à travers par exemple l'étude du fonctionnement de la reformulation des communiqués de presse ou de la réécriture d'entretiens oraux. Il est apparu dans cette étude que ce discours autre est toujours présent dans le discours journalistique, créant une hétérogénéité linguistique au sein de ce discours, et que seuls des critères culturels permettent de retrouver une cohérence entre les deux discours, ici à travers le critère d'objectivité et la recherche de légitimation. Il serait intéressant de poursuivre cette étude des valeurs journalistiques en nous demandant notamment dans quelle mesure le discours journalistique serait le produit d'un système de valeurs propres à une certaine communauté culturelle. 


\section{BIBLIOGRAPHIE}

Adam, Jean-Michel et Gilles Lugrin. 2006. «Effacement énonciatif et diffraction co-textuelle de la prise en charge des énoncés dans les hyperstructures journalistiques ». Semen 22, Énonciation et responsabilité dans les médias [en ligne]. Consulté le 17 août 2009. <http://semen.revues.org/ 4381.htm>.

Authier-Revuz, Jacqueline. 1996. "Remarques sur la catégorie de l'îlot textuel' ». Cahiers du français contemporain 3, Hétérogénéité en discours, 91-115.

Authier-Revuz, Jacqueline. 2004. « La représentation du discours autre : un champ multiplement hétérogène ». In Lopez Munoz J. M., S. Marnette et L. Rosier (dir.), Le Discours rapporté dans tous ses états. Paris : L'Harmattan, 35-53.

Charaudeau, Patrick. 2005. Les médias et l'information. L'impossible transparence du discours. Bruxelles : de Boeck-INA.

Charaudeau, Patrick. 2006. « Discours journalistique et positionnements énonciatifs. Frontières et dérives ». Semen 22, Énonciation et responsabilité dans les médias [en ligne]. Consulté le 17 août 2009. <http://semen.revues.org/document2793.html>.

Charaudeau, Patrick et Dominique Maingueneau. 2002. Dictionnaire d'analyse du discours. Paris : Seuil.

Komur, Greta. 2004. «L'îlot textuel et la prise de distance par le locuteur dans le genre journalistique ». In Lopez Munoz J. M., S. Marnette et L. Rosier (dir.), Le Discours rapporté dans tous ses états. Paris :L'Harmattan, 54-64.

Krieg-Planque, Alice. 2006. « 'Formules' et 'lieux discursifs' : propositions pour l'analyse du discours politique ». Semen 21, Catégories pour l'analyse du discours politique [en ligne]. Consulté le 17 août 2009. <http://semen.revues.org/1938.html>.

Lorda, Clara-Ubaldina. 2001. « Les articles dits d'information : la relation de déclarations politiques ». Semen 13, Genres de la presse écrite et analyse de discours [en ligne]. Consulté le 3 décembre 2009. <http://semen.revues.org/2625.htm>.

Moirand, Sophie. 2007. Le discours de la presse quotidienne : observer, analyser, comprendre. Paris : Presses Universitaires de France.

Pennec, Blandine. 2010. «L'expression de la cause en anglais journalistique : essai de caractérisation, à travers l'exemple du Financial Times ». ASp 57, 31-47.

Rey-Debove, Josette. 1997. Le Métalangage. Paris : Armand Colin-Masson.

Vignaux, Georges, 1981. «Énoncer, argumenter : opérations du discours, logique du discours ». Langue française 50, 91-116.

The Wall Street Journal Media Kit, Global Readership. 2010. Consulté le 17 août 2009. <http:// wsjmediakit.com/magazine/audience/profileglobal>.

\section{ANNEXES}

\section{Annexe 1}

Liste des articles : codes et titres 
Chicago Tribune

CT1: President keeps his options open for compromise

CT2: The New "New Deal"

CT3: Hope smokes eternal when it's China-grown

Los Angeles Times

LAT1 : Dollars and sense

LAT2 : Major points of President Obama's speech

LAT3 : How would Obama's proposed spending 'trigger' work?

LAT4 : Obama's healthcare speech helps unify Democrats

LAT5 : Outrage over Rep. Joe Wilson's outburst isn't dying down

The New York Times

NYT1 : Obama's Health Care Plan Builds on Others' Ideas

NYT2 : Obama, Armed With Details, Says Health Plan Is Necessary

NYT3 : In Lawmaker's Outburst, a Rare Breach of Protocol

NYT4 : On Brink, Obama Is Resolute and Clear

NYT5 : Aim of Obama Health Speech: Reigniting a Presidency

NYT6 : Check Point: Examining Obama's Assertions

NYT7 : Democrats Seem Unified by Obama's Speech

NYT8 : Automatic Cuts Could Help Push Past a Health Hurdle

NYT9 : In Heckler's District, Support for the Outburst

NYT10 : President's Speech Allays Some Fears in the Health Insurance Industry

The Wall Street Journal

WSJ1 : Emotions Run High in Capitol

WSJ2 : Americans, While Sympathetic, Worry About Cost

WSJ3 : An Appeal to the Center - and to Activists on the Left

WSJ4 : Obama Doubles Down

WSJ5 : Small Businesses Wary of Health Reform Costs

WSJ6 : President Makes His Pitch

WSJ7 : Medicare for Dummies

WSJ8 : 'You Lie!' Jars Washington but Resonates Back Home

The Washington Post

WP1 : With His Top Priority on the Line, President Reframes Critical Debate

WP2 : In N. Virginia Exurb, the Holdouts Are Unmoved

WP3 : Obama Implores Congress to Act; In Arguing for a Public option, He emphasizes

It's 'Only One Part of My Plan'

WP4 : Details Still Lacking On Obama Proposal; White House Unclear on How Some Far-

Reaching Goals Would Be Met

WP5 : On Malpractice Reform, Fine Print Is Still Hazy; Obama Pitches State-Level

Experiments to Reduce Litigation but Offers Few Details

\section{NOTES}

1. Retranscription disponible sur <http://www.whitehouse.gov/the_press_office/Remarks-bythe-President-to-a-Joint-Session-of-Congress-on-Health-Care/> 
2. Le code des articles correspond au journal dont ils sont tirés; CT : Chicago Tribune, LAT : Los Angeles Times, NYT : New York Times, WSJ : Wall Street Journal et WP : Washington Post. Le titre des articles est fourni en annexe.

3. Le discours de B. Obama contient un total de 5563 mots, les 57 occurrences de I représentent donc $10,2 \%$ de ce total.

4. Les proportions correspondant aux nombres d'occurrences (colonnes 1 et 2) sont exprimées en pour mille sur la totalité des mots du texte concerné (colonne 5), tandis que la proportion d'associations avec un certain type de procès (colonne 4) est exprimée en pourcentage du nombre d'occurrences des mots (colonnes 1 et 2). Par exemple, les vingt-cinq occurrences d'associations avec des verbes exprimant la volonté représentent vingt pour cent de la somme des I et des we relevés.

5. D'après les données de 2010 sur le site Internet du Wall Street Journal, ses lecteurs ont un revenu moyen par foyer 298690 dollars, revenu moyen plus élevé que celui des lecteurs des autres journaux.

\section{RÉSUMÉS}

Cet article présente une analyse du rôle du discours rapporté dans trente et un articles de presse à propos du discours prononcé par le président Obama devant le Congrès en septembre 2009 ; le corpus est tiré de cinq journaux différents. L'auteur a tenté de comprendre les différences entre le discours utilisé dans les citations, celles-ci émanant aussi bien de B. Obama que d'autres sources commentant le discours, et le discours produit directement par le journaliste, ainsi que la manière dont le discours rapporté est intégré dans les articles. Il apparaît que le journaliste sélectionne certaines formes de discours rapporté (discours direct ou combinaisons de formes), au détriment d'autres formes (discours indirect), et que les citations sont généralement plus personnalisées que le texte encadrant, ce qui permet au journaliste d'exprimer des opinions sans le faire en son nom propre. Malgré ces différences, l'auteur suggère que l'utilisation du discours rapporté est conforme à certaines conventions journalistiques telles que l'objectivité ou la légitimation du discours.

This paper presents an analysis of the role of reported speech in newspaper articles commenting on a political speech. The corpus comprises thirty-one articles published in five American newspapers and dealing with President Obama's speech on the healthcare reform before Congress in September 2009. The aim is to understand the differences between the discourse used in quotes, both from Obama's speech and from other sources commenting on the speech, and the discourse produced directly by the journalists. Results show that journalists select some forms of reported speech such as direct speech or combined forms, and tend to exclude others, such as indirect speech, and that the quotes are much more personalized than the rest of the articles, which allows the journalists to express opinions they could not express directly. Despite these differences, it is argued that the use of reported speech is consistent with the respect of certain press conventions such as objectivity and the legitimization of the journalist's discourse. 
INDEX

Mots-clés : discours journalistique, discours rapporté, journalisme politique, légitimation, objectivité

Keywords : legitimization, news discourse, objectivity, political journalism, reported speech

\section{AUTEUR}

\section{CAROLINE PEYNAUD}

Caroline Peynaud est agrégée d'anglais, enseignante dans le secondaire et doctorante à l'Université Bordeaux Segalen, où elle est membre de l'EA 4140 (ASPDA). Ses recherches portent sur une caractérisation discursive et culturelle du milieu journalistique aux États-Unis. caroline.peynaud@free.fr 